\title{
Establishment of Local Diagnostic Reference Levels in Conventional Radiography: A Pilot Study in Dakar, Senegal
}

\author{
Adji Yaram Diop ${ }^{1,2}$, Magatte Diagne2, Ndeye Arame Boye Faye ${ }^{1,3}$, Mamadou Moustapha Dieng1,2 \\ ${ }^{1}$ Cheikh Anta Diop University of Dakar, Dakar, Senegal \\ ${ }^{2}$ Aristide Le Dantec Hospital, Joliot Curie Institute of Cancer, Dakar, Senegal \\ ${ }^{3}$ Nuclear Radiation Protection and Safety Authority, Dakar, Senegal \\ Email: adjiyaram.diop@ucad.edu.sn
}

How to cite this paper: Diop, A.Y., Diagne, M., Boye Faye, Nd.A. and Dieng, M.M. (2022) Establishment of Local Diagnostic Reference Levels in Conventional Radiography: A Pilot Study in Dakar, Senegal. World Journal of Nuclear Science and Technology, 12, 28-42.

https://doi.org/10.4236/wjnst.2022.121004

Received: November 28, 2021

Accepted: January 23, 2022

Published: January 26, 2022

Copyright $\odot 2022$ by author(s) and Scientific Research Publishing Inc. This work is licensed under the Creative Commons Attribution International License (CC BY 4.0).

http://creativecommons.org/licenses/by/4.0/

\begin{abstract}
Diagnostic Reference Levels (DRLs) are indicators that allow assessing the quality of equipment and procedures from the point of view of the doses delivered to patients and subsequently initiate corrective actions if necessary. The purpose of this study is to encourage health professionals to investigate patient radiation doses and to determine whether those doses comply with the principles of radiation protection in medical fields so as to improve practices by reducing patient exposure without reducing clinical effectiveness. To perform this work, we have investigated patient doses for different radiological examinations from six (6) medical centers in Dakar, including the following nine routine types: chest (PA), abdomen (AP), pelvis (AP), cervical spine (AP), lumbar spine (AP, Lat), hip (AP), thoracic spine (AP, Lat). Three types of data were collected, i.e., X-ray tube machine data, patient data and output measurements. The data were analyzed statistically and the median, minimum, maximum, and third quartile values were calculated and displayed throughout boxplots graphs for all exams and medical centers. The two sigma range (95\% confidence interval) was also checked. Comparison of third quartiles of Entrance Surface Dose (ESD) and Dose Area Product (DAP) by type of examination with recommended international DRLs was performed. The third quartile of ESD for pelvis (AP) and thoracic spine (AP) was up to $16 \%$ and 38\% higher, respectively than their corresponding DRLs in the European Commission Report RP 180 Part 2. For all exams, except thoracic spine (lat), the third quartiles of the dose area product were higher than the corresponding DRLs in the above report. The source of dose variability between medical centers was related to many parameters such as poor radiographic techniques, lack of modern X-ray machines and adequately documented radiation
\end{abstract}


protection practices. The results show the need to develop protocols for dose measurement as well as to carry out quality assurance programs and dose optimization in Senegal.

\section{Keywords}

Entrance Surface Dose, Diagnostic Radiology, Dose Area Product, Optimization, X-Rays

\section{Introduction}

Diagnostic X-rays represent the major portion of radiation exposure from artificial origin to the population. According to the World Health Organization (WHO), more than 3.6 billion diagnostic radiology exams are performed every year around the world. X-ray examinations are an established tool of medical diagnosis and patients can undoubtedly obtain enormous benefits from these examinations. However, the ionizing nature of the X-rays means that their use is not entirely without risk. The stochastic effect of low doses from ionizing radiation follows a linear model without threshold and at a long term. For this reason, all exposures to diagnostic X-rays need to be justified and optimized in terms of benefit and risk [1]. These effects are not well known, that is why it is important to monitor patient dose.

The International Commission on Radiological Protection (ICRP) suggested general principles of radiation protection: justification, optimization and dose limit. In medical exposure, dose limits are not at all relevant since ionizing radiation, used at the appropriate level of dose for a particular medical purpose, is an essential tool that will cause more good than harm. Therefore medical radiation has no dose limits, and generally uses diagnostic reference level (DRL) as a reference value for optimization of practice [1]. The goal of the optimization process is to provide an acceptable image quality by keeping the corresponding radiation dose As Low As Reasonably Achievable (ALARA) [2]. In 1997, the ICRP introduced DRLs with the goal to reduce the unnecessary radiation exposure by setting given thresholds [3]. DRLs have been defined in European Commission's legislation (EC, 1997) as dose levels in medical radiodiagnostic practices in the case of radiopharmaceuticals levels of activity for typical examination for group of standard patients or standard phantoms for broadly defined type of equipment. The International Atomic Energy Agency (IAEA) through the International Action Plan on Radiation Protection of Patients and the ICRP have for some time carried out important efforts to ensure that in the medical applications of the ionising radiations, the optimisation of radiological protection of patients is fundamental, to such a point that they include it directly as a requirement for these practices (in the International Basic Safety Standards for Protection against Ionising Radiation and for the Safety of Radiation Sources (BSS)-GSR Part 3, 2014). 
The reference value represents an efficient tool for radiation protection of patients and dose optimization in diagnostic procedures and allows identifying the situation where the level of patient dose is unusually high [4]. The council of the European Union has adopted the concept of DRLs in the Medical Exposure Directive 2013/59/Euratom of December 5, 2013 laying down basic safety standards for protection against the dangers arising from exposure to ionising radiation, and repealing Directives 89/618/Euratom, 90/641/Euratom, 96/29/Euratom, 97/43/Euratom and 2003/122/Euratom. The member states of the EU are obliged to adopt the DRLs into national legislation and regulation concerning radiation protection [5] [6]. Many countries and legal institutions use DRLs in radiological investigations which serve as a guide for patient dose reduction. In Africa, many countries started to establish their own DRLs as suggested by ICRP. Konate et al. (2019) reported the DRLs in frontal chest X-rays in western Côte d'Ivoire. In 2017, Moifo et al. established in a pilot study DRLs of adults CT in Cameroon [7] [8]. The reference value corresponds to the $3^{\text {rd }}$ quartile $\left(75^{\text {th }}\right.$ percentile) value of the distribution of median values from all the examination, 75\% of individual receive doses less than this value. DRLs provide a convenient system that allows hospitals (X-ray departments) to compare the radiation doses delivered to the patients. For that purpose, the dose should be expressed in terms of quantities that are clearly defined and can be easily measured directly or calculated from readily available exposure parameters [9]. The dosimetric parameter should bear a nearly linear relationship with radiation risks associated with examinations. To achieve these objectives the following dosimetric parameters have been widely adopted for monitoring in conventional radiography:

1) Entrance surface dose (ESD) - conventional radiography (could be obtained with TLD or by calculations);

2) Dose-area product (DAP)—conventional radiography (obtainable with DAP meter or by calculations).

These dosimetric parameters were introduced to verify that the dose descriptors used during imaging process are below the defined European values established after many trials. In Senegal, both local and national DRLs (LDRLs and NDRLs, respectively) are not available.

Registration of the existing X-ray units using conventional radiography, CT, fluoroscopy etc. is currently being undertaken throughout Senegal. In addition, a National Radiation Dose Database (NRDD) is required to access dose information and facilitate processing for future optimization programs. To establish patient DRLs for various radiography tests and raise public awareness about patient dose, it is useful to identify the medical centers associated with higher radiation doses. As a result, the adoption of measures such as equipment quality control can lead to a reduction in patient doses while improving image quality [10].

The lack of radiation protection culture and patient's radiation protection practices, which are poorly documented in Senegal, make a detailed study very timely. The one presented here is a first that aims at examining the situation in 
six public hospitals in Dakar so as to evaluate how the optimization principle of the ICRP could be put into practice. The intention of this paper is to establish the dose references in conventional radiology for adult patients, for the purposes of determining whether these doses comply with the requirements of the BSS and of improving practices, in order to minimise the dose received by the patients.

\section{Materials and Methods}

This study began after we received permission from each of the managers of the six (6) medical centers, i.e., Hopital Aristide Le Dantec (HALD), Centre Hospitalier National Universitaire de Fann (CHNUF), Institut d'Hygiène Social (IHS), Hopital Général de Grand Yoff (HOGGY), Centre Hospitalier National de Pikine (CHNP), Service d'Aide Medicale d'Urgence (SAMU). The scope of the dose survey and the measurements were limited to the most frequent X-ray examinations that give a large contribution to the collective dose. The following nine routine types of $\mathrm{X}$-ray examinations are included in the investigations: chest (PA), abdomen (AP), pelvis (AP), hip (AP), cervical spine (AP), thoracic spine (AP), thoracic spine (LAT), lumbar spine (AP), and lumbar spine (LAT). Chest (PA), Abdomen (AP) and cervical spine (AP) examinations were acquired in standing position while the other examinations were taken in supine position. The patients that underwent AP/PA and LAT projection were considered as separate cases to estimate the dose for each projection. The data were collected by the physicist operating in the rooms included in the survey. The exposure parameters displayed on the console of the $\mathrm{X}$ ray unit during examination were recorded by the physicist. The examinations were performed in seven radiographic rooms in total, all of them equipped with systems using AGFA Computed Radiography (CR) with Automatic Exposure Control (AEC). The X-ray equipment was checked through quality control program to ensure the consistency of the equipment performance, the reliability and reproducibility of the exposure parameters. The quality control tests included the tube voltage accuracy and reproducibility, the current time product (mAs) linearity, the Half Value Layer (HVL) and the X-ray tube output measurement. The latter was measured at a distance of $100 \mathrm{~cm}$ from the X-ray source to the chamber (detector), for a tube voltage between 45 and $130 \mathrm{kVp}$ in incremental steps of $5 \mathrm{kVp}$. All measurements were performed using Xi model R/F, serial number 185512 produced by Unifors RaySafe, Sweden. This is a multi-parameter X-ray detector which can measure $\mathrm{kVp}$, dose, dose rate, HVL and time. To ensure the accuracy and precision of the quantities derived from calculation, we performed dosimetric measurements based on the recorded parameters used during examination.

Details on 2217 X-ray examinations were collected during a period of one year, and at least 30 patients were observed for each examination type. All the nine exams were not performed in each room. For each examination, personal data and technical parameters were collected according to a questionnaire designed for the patient's dosimetry protocol as follows: 
- Radiological room and equipment;

- Patient sex, age, weight, height, thickness;

- Type of procedure;

- Examination parameters: kVp, mAs, Film (cassette AGFA) size and Focus-Film Distance (FFD).

The knowledge of tube output, tube voltage, tube current, exposure time and Focus-Skin Distance (FSD) enables the deduction of the air kerma at the point corresponding to the position of the patient's skin. The FSD was calculated for each examination by subtracting the patient thickness and film-table top from the FFD. The air kerma measured in the primary X-ray beam in the entrance plane of the patient allowed to evaluate the entrance surface dose (ESD) which is closely related to the backscattering factor. The latter is defined as the ratio of the air kerma on the surface of the phantom and air kerma free in air [11]. Typical backscattering values for diagnostic X-ray beam qualities range from 1.25 to 1.55. It is taken as 1.35 for adult patients [12] [13]. The ESD represents the absorbed dose at the patient entrance and is given by Equation (1). For each patient, it was calculated using real examination data.

$$
\mathrm{ESD}=\mathrm{BSF} \times \text { Output }_{D} \times \mathrm{mAs} \times 100^{2} /\left[\mathrm{FFD}-(a+b)^{2}\right]
$$

In this equation, BSF is the backscatter factor, $a$ is the patient thickness, $b$ is the distance between the top of the table and the film and mAs is the tube current time product [14]. The X-ray tube output of each system was obtained during quality control with this power function:

$$
\operatorname{Output}(\mu \mathrm{Gy} / \mathrm{mAs})=A(\mathrm{kVp})^{n}
$$

In this equation, $A$ is the fitting factor and $n$ is the power, both derived from the plotting of the tube output measurement against the tube voltage [15].

The dose area product (DAP), which is the product of the Incidence Air Kerma (IAK) (i.e. ESD in the absence of backscattering) by the irradiated area, is not only a quick and simple measurement but also a valuable radiation dose descriptor. Its advantage is that the biological effects of radiation are dependent on the radiation dose and the irradiated area of the body. The DAP is also applicable for quality assurance and functional analysis of X-ray machines [16] [17] [18]. It was calculated based on this formula [19]:

$$
\mathrm{DAP}=\mathrm{IAK} \times \text { Area }
$$

The local DRLs were estimated at the 75th percentile of ESD and DAP for each exam and room to further assist in the optimization process by providing a local comparator linked to the differences between technology and variations in the radiographic technics utilized by the various radiographers as well as the lack of national DRLs in the country. The global DRLs also for all the exams were defined at the 75th percentile in terms of Entrance Surface Dose (ESD) and Dose Area Product (DAP) distributions [20]. Microsoft Excel software was used to handle the data, descriptive statistics (median, mean, standard deviation and 
range of value) were performed for expressing values of patients data (age, weight), exposure parameters (FSD, $\mathrm{kVp}, \mathrm{mAs}$ ) but also dose indicators (ESD and DAP). The statistical software $\mathrm{R}$ was used to evaluate the 3rd quartile of ESD and DAP. Figure 1 shows a flowchart listing the steps leading to the establishment of the LDRLs in conventional radiography in six medical centers in Dakar.

\section{Results}

To obtain a typical dose estimate in a typical patient, the measurements were performed on a representative sample of adult patients. 2217 patients were included in this study. Table 1 shows the specific data of the X-ray units investigated along with tolerance values from the quality control performed on the equipment before data collection. The quality control (QC) checks done on the $\mathrm{X}$-ray equipment compared with the recommended tolerances revealed that all the X-ray machines used in the study passed the entire range of the QC test performed.

The patient data (age, weight) as well as exposure parameters $(\mathrm{kVp}, \mathrm{mAs}$ and FSD) classified per examination have been summarized in Table 2. The patients were 1063 men and 1154 women aged between 18 and 97 years (mean age of 48 years). The knowledge of the X-ray tube output measured during quality control test at a distance of $100 \mathrm{~cm}$ with fixed $\mathrm{mAs}$ allowed the calculation of the dose received by the patients as shown in Figure 2. A power law equation if obtained for the variation and replacing the unknown by the value of the tube voltage leads to the X-ray tube output for that voltage.

We firstly present the results in term of entrance surface dose (ESD) and dose area product (DAP) individually as boxplot graphs (Figure 3 and Figure 4) in which each hospital is represented for all the examinations. The median, the minimum, the maximum, the interquartile range and the outliers are shown.

The histograms of ESD and DAP values for all examinations are presented in Figure 5 and Figure 6. Most of the ESD and DAP values show a positively

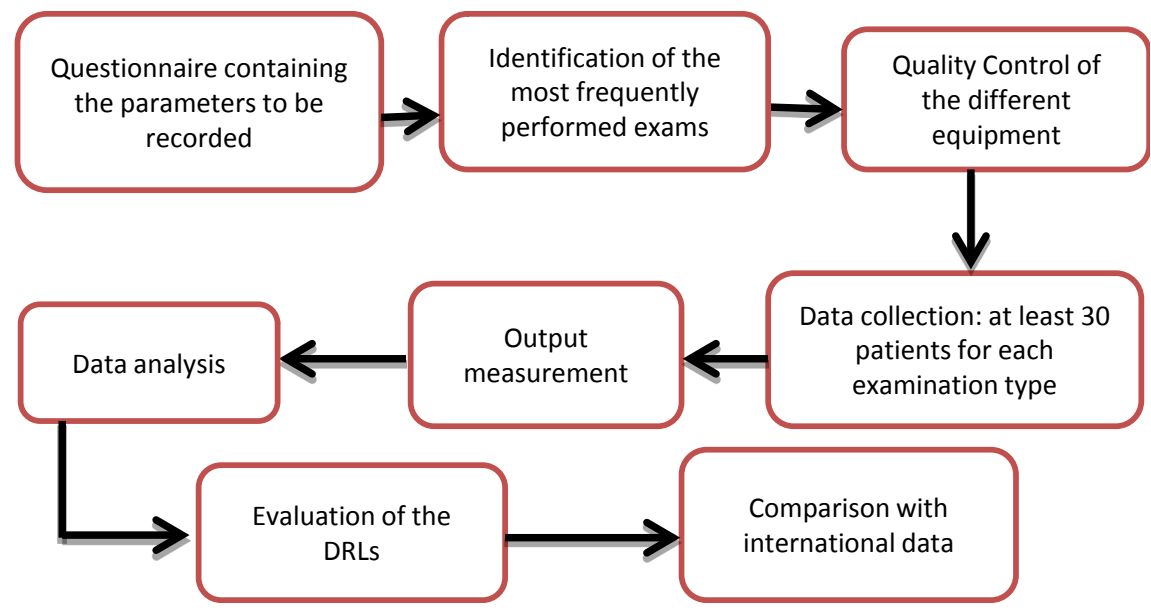

Figure 1. Steps of the process leading to the establishment of the LDRLs in conventional radiography in six medical centers in Dakar. 


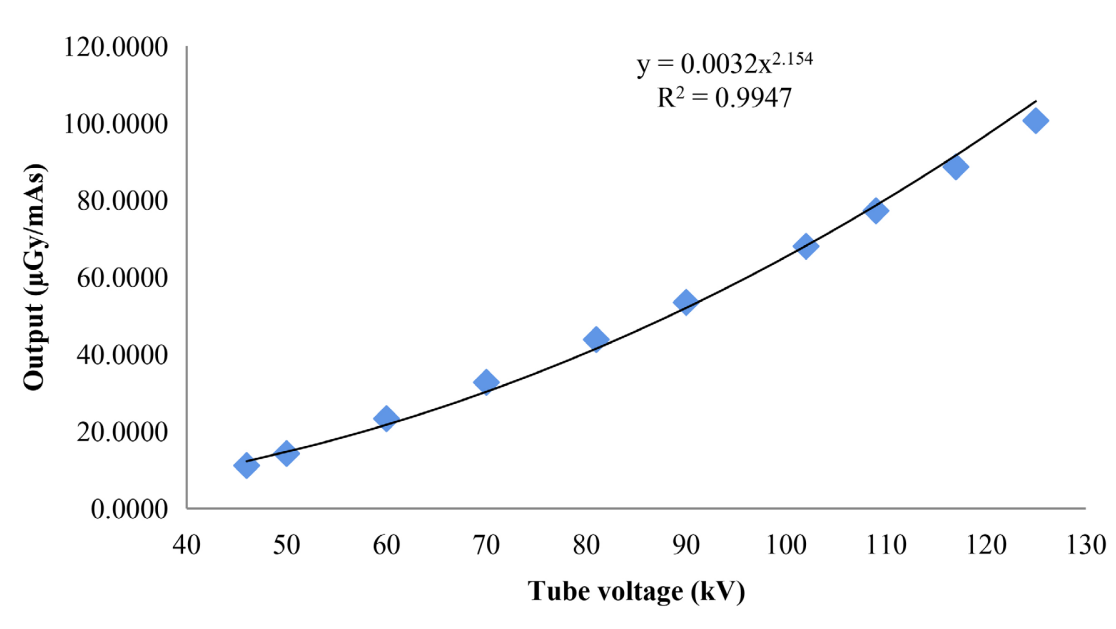

Figure 2. X-ray tube output curve. These measurements were done at $1 \mathrm{~m}$ from the source with a tube voltage varying from 45 to $130 \mathrm{kVp}$ in incremental steps of $5 \mathrm{kVp}$.
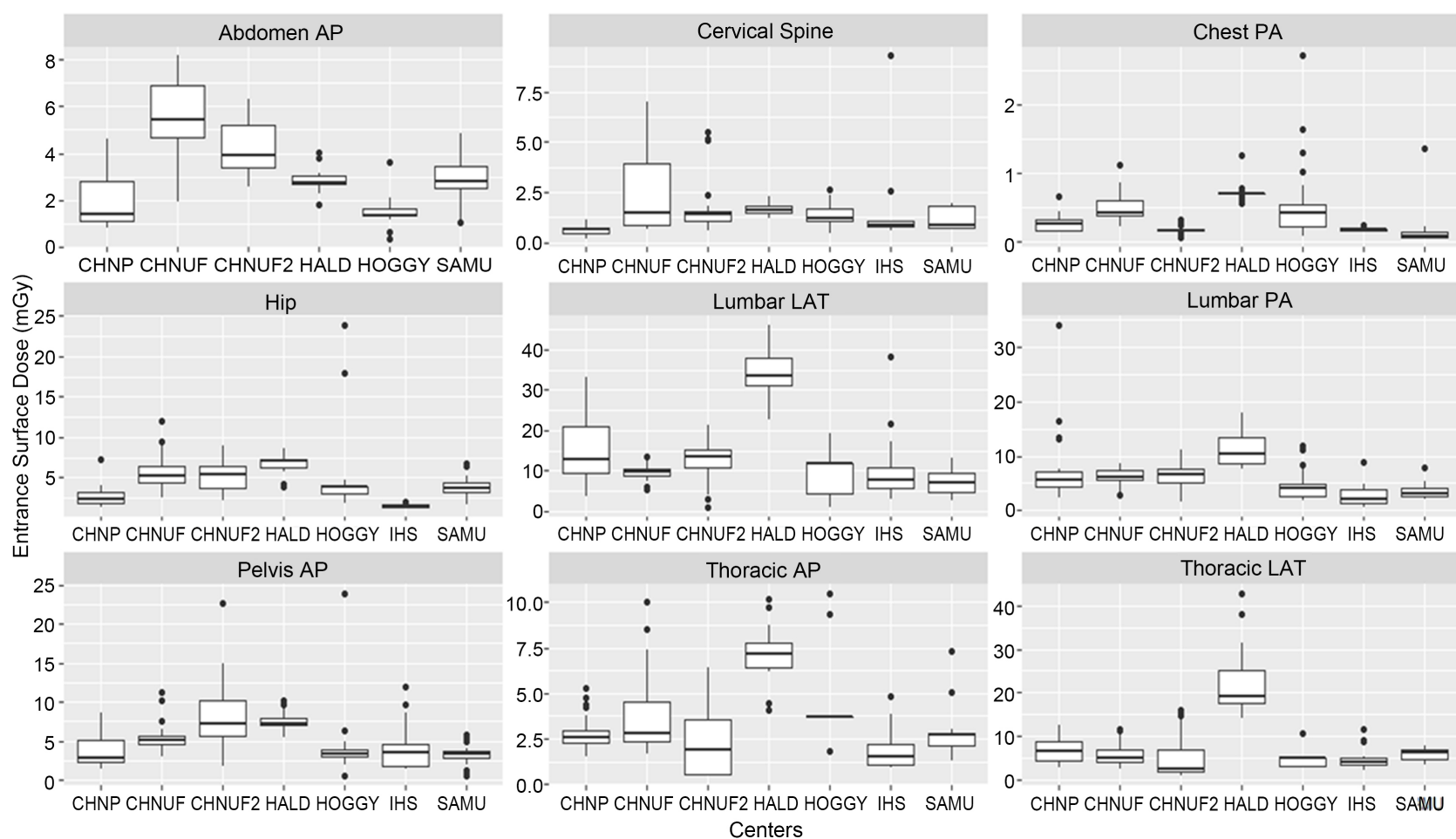

CHINP CHNUEF CHNUUF2 HÁLD HOGGY IH'S SAMUU

Figure 3. Comparative results of the different medical centers and examination in term of ESD. The values are the dose (mGy) at the patient's entrance surface obtained by calculation with the examination parameters.

Table 1. Specific data of the X-ray units investigated together with tolerance values. We performed QC test in each room for the six medical centers and compared the results with the tolerance values.

\begin{tabular}{|c|c|c|c|c|c|c|}
\hline \multirow{3}{*}{ Hospitals } & \multicolumn{6}{|c|}{ Quality Control } \\
\hline & $\mathrm{kVp}$ & $\mathrm{kVp}$ & Dose & mAs & $\mathrm{HVL}$ at $80 \mathrm{kV}_{\mathrm{l}}$ & Tube Yield At $80 \mathrm{kVp}$ \\
\hline & Accuracy (\%) & Reproductibility (\%) & Reproductibility (\%) & reproductibility (\%) & $(\mathrm{mmAl})$ & $(\mathrm{mGy} / \mathrm{mAs})$ at $1 \mathrm{~m}$ \\
\hline HALD & M & 0.52 & 0.35 & 0.28 & 3.10 & 0.077 \\
\hline HIS & 9.34 & 1.00 & 0.84 & 5.08 & 3.30 & 0.043 \\
\hline CHNP & 1.34 & 0.40 & 0.35 & 1.11 & 3.22 & 0.060 \\
\hline
\end{tabular}


Continued

\begin{tabular}{ccccccc}
\hline & 5.58 & 0.22 & 1.42 & 4.82 & 3.82 & 0.030 \\
HOGGY & 2.96 & 3.02 & 0.69 & 5.45 & 3.01 & 0.049 \\
CHNUF & 1.11 & 0.20 & 0.28 & 0.57 & 5.13 & 0.027 \\
CHNUF2 & 1.73 & 0.32 & 0.09 & 1.40 & 3.45 & 0.040 \\
SAMU & 0.48 & 0.57 & 0.39 & 0.73 & 3.72 & 0.049 \\
Tolerance & Dev $<10 \%$ & $\mathrm{CV}<5 \%$ & $\mathrm{CV}<20 \%$ & $\mathrm{CV}<10 \%$ & $>2.50 \mathrm{mmAl}$ & $>0.025 \mathrm{mGy} / \mathrm{mAs}$ \\
\hline
\end{tabular}

Table 2. Summary of patients' characteristics and examination parameters. We report here the mean value and the corresponding range (minimum - maximum).

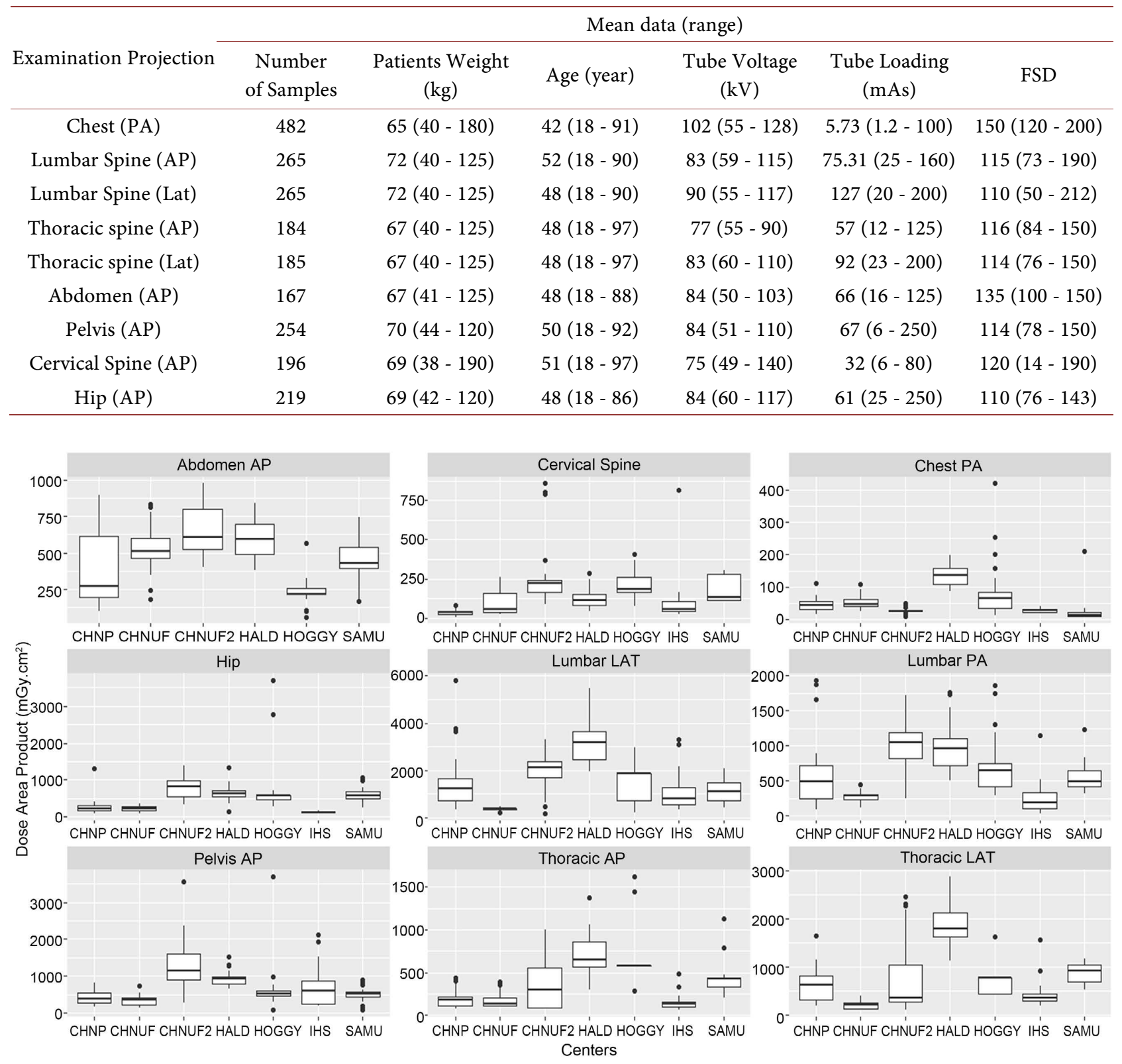

Figure 4. Comparative results of the different medical centers and examination in term of DAP. The values are the dose (in $\mathrm{mGy} \cdot \mathrm{cm}^{2}$ ) obtained by the product of the IAK (i.e., ESD without backscattering) by the area in concern. 

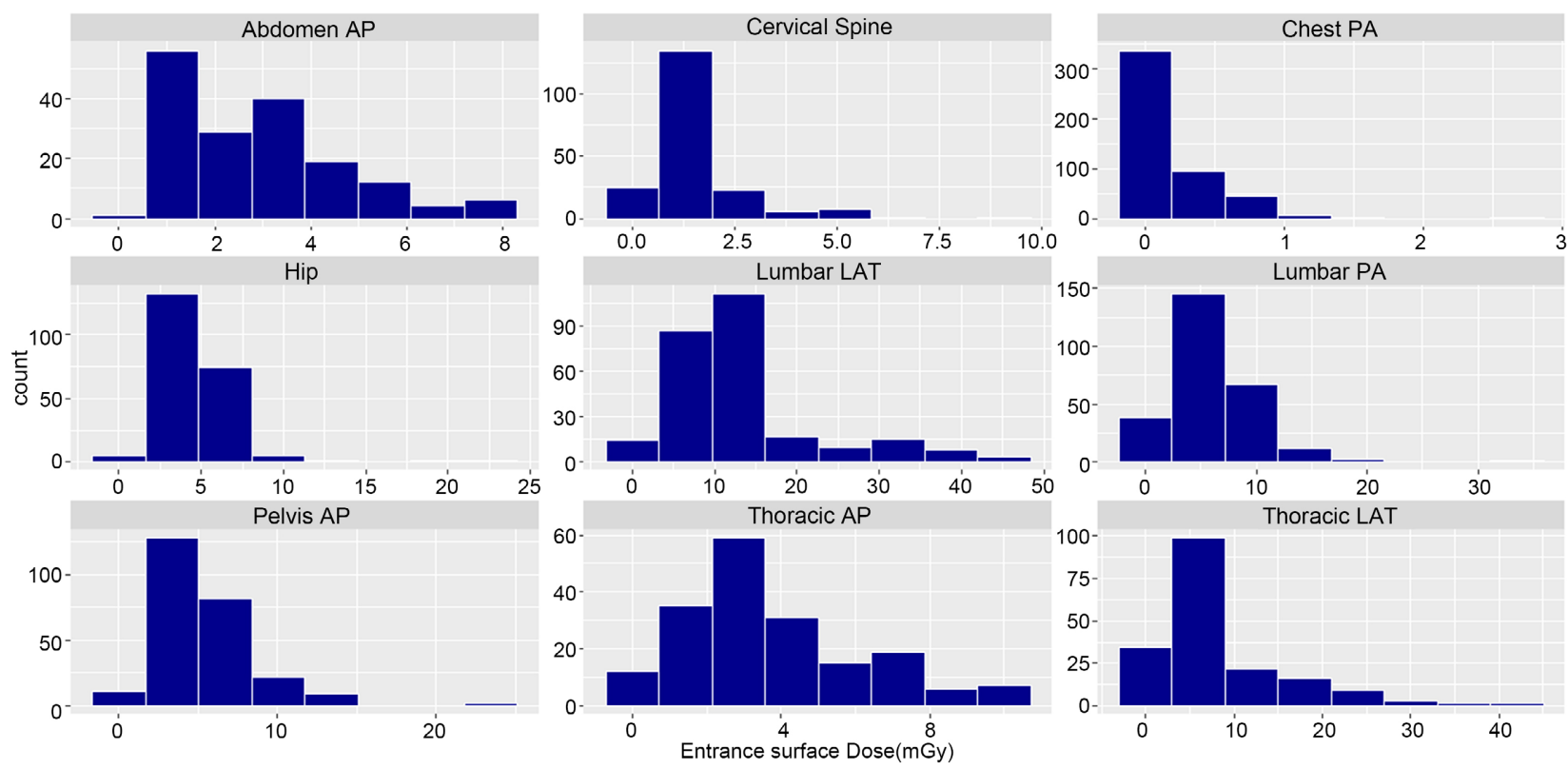

Figure 5. Histogram of the distribution of median ESD (in $\mathrm{mGy}$ ) values resulting from dosimetric evaluations carried out for all medical centers by examination projection.
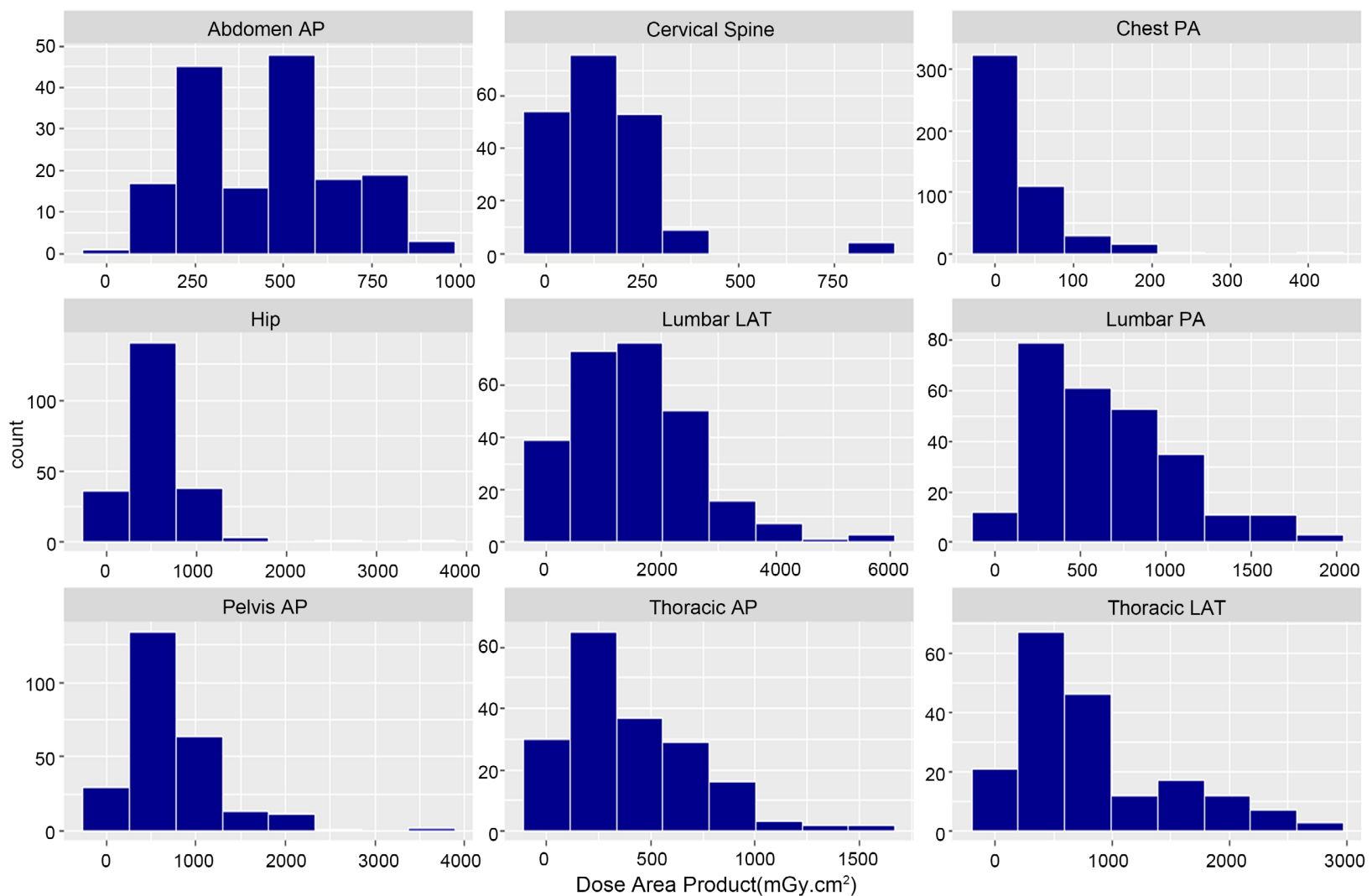

Figure 6. Histogram of the distribution of median DAP (in $\mathrm{mGy} \cdot \mathrm{cm}^{2}$ ) values resulting from dosimetric evaluations carried out for all medical centers by examination projection.

skewed distribution and in this case the $95 \%$ confidence interval (CI) values are better defined than the maximum and minimum values. The median, standard 
deviation and 95\% CI for both ESD and DAP and also globally are presented in Table 3. The 75th percentiles of ESD and DAP values for each exam were compared with recommended international DRLs for some European countries in Table 4 and Table 5.

Table 3. ESD and DAP values obtained from X-ray examination for all medical centers.

\begin{tabular}{|c|c|c|c|c|c|c|}
\hline \multirow[b]{2}{*}{ Examination Projection } & \multicolumn{3}{|c|}{ ESD (mGy) } & \multicolumn{3}{|c|}{$\mathrm{DAP}\left(\mathrm{cGy} \cdot \mathrm{cm}^{2}\right)$} \\
\hline & Median & Standard deviation & $\begin{array}{l}\text { 95\% Confidence } \\
\text { Interval }\end{array}$ & Median & Standard deviation & $\begin{array}{c}\text { 95\% Confidence } \\
\text { Interval }\end{array}$ \\
\hline Chest (PA) & 0.16 & 0.3 & $0.23-0.27$ & 24.96 & 41.6 & $35.35-42.7$ \\
\hline Lumbar Spine (AP) & 5.10 & 4.0 & $5.41-6.28$ & 617.30 & 417.4 & $612.94-713.44$ \\
\hline Lumbar Spine (Lat) & 13.00 & 9.0 & $12.14-14.35$ & 1481.01 & 1044.6 & $1413.12-1664.65$ \\
\hline Thoracic spine (AP) & 2.81 & 2.0 & $3.36-4.06$ & 328.45 & 309.1 & $347.26-436.58$ \\
\hline Thoracic spine (Lat) & 5.22 & 7.0 & $8.08-9.15$ & 640.64 & 663.6 & $721.3-912.55$ \\
\hline Abdomen (AP) & 2.68 & 2.0 & $2.73-3.27$ & 474.75 & 218.2 & $410.38-476.57$ \\
\hline Pelvis (AP) & 4.69 & 3.0 & $4.98-5.77$ & 592.41 & 518.6 & $672.46-800.01$ \\
\hline Cervical Spine (AP) & 1.22 & 1.0 & $1.37-1.72$ & 123.68 & 13.6 & $132.55-170.23$ \\
\hline Hip (AP) & 4.01 & 2.0 & $4.39-5.04$ & 584.33 & 378.1 & $525.18-625.32$ \\
\hline
\end{tabular}

Table 4. Comparison of $75^{\text {th }}$ percentiles with the literature for each examination in term of ESD.

\begin{tabular}{cccccccc}
\hline & \multicolumn{7}{c}{ ESD (DRLs) } \\
\cline { 2 - 8 } $\begin{array}{c}\text { Examinations } \\
\text { Projection }\end{array}$ & $\begin{array}{c}\text { Our } \\
\text { study }\end{array}$ & $\begin{array}{c}\text { France } \\
{[21]}\end{array}$ & UK [20] & $\begin{array}{c}\text { Germany } \\
{[22]}\end{array}$ & $\begin{array}{c}\text { Sweden } \\
{[23]}\end{array}$ & Italy [15] & $\begin{array}{c}\text { DRLs } \\
{[24]}\end{array}$ \\
\hline Chest (PA) & 0.3 & 0.3 & 0.15 & 0.3 & 0.3 & 0.4 & 0.3 \\
Lumbar Spine (AP) & 7.5 & 10 & 5 & 10 & 10 & 10 & 10 \\
Lumbar Spine (Lat) & 15.2 & 30 & 11 & 30 & 30 & 30 & 25 \\
Thoracic spine (AP) & 5.1 & 10 & 4 & 10 & & 10 & 8 \\
Thoracic spine (Lat) & 10.5 & 10 & 4 & 10 & 10 & 10 & 9 \\
Abdomen (AP) & 3.8 & 5 & 2 & 5 & 5 & 5 & 4 \\
Pelvis (AP) & 6.9 & - & - & - & - & - & 5 \\
Cervical Spine (AP) & 1.7 & - & - & - & - & - & 7 \\
Hip (AP) & 6.1 & - & - & - & - & - & 9 \\
\hline
\end{tabular}

Table 5. Comparison of $75^{\text {th }}$ percentiles with the literature for each examination in term of DAP.

\begin{tabular}{cccccccc}
\hline & \multicolumn{7}{c}{ DAP (DRLs) } \\
\cline { 2 - 8 } $\begin{array}{c}\text { Examinations } \\
\text { Projection }\end{array}$ & $\begin{array}{c}\text { Our } \\
\text { study }\end{array}$ & $\begin{array}{c}\text { France } \\
{[21]}\end{array}$ & UK [20] & Germany & Swiss & Sweden & DRLs \\
{$[22]$} & {$[25]$} & {$[23]$} & {$[24]$} \\
\hline Chest (PA) & 37.0 & 25 & 11 & 20 & 20 & 60 & 25 \\
\hline
\end{tabular}




\begin{tabular}{cccccccc} 
Continued \\
\hline Lumbar Spine (AP) & 913.7 & 450 & 160 & 320 & 320 & 1000 & 450 \\
Lumbar Spine (Lat) & 2139.9 & 800 & 250 & 800 & 800 & 1000 & 800 \\
Abdomen (AP) & 575.9 & 550 & 260 & 550 & - & - & 700 \\
Pelvis (AP) & 1130.5 & 600 & 210 & 500 & 500 & 400 & 700 \\
Cervical Spine (AP) & 586.4 & 41 & 80 & 110 & 110 & - & 75 \\
Thoracic Spine (AP) & 918.0 & 110 & - & - & - & - & 175 \\
Thoracic Spine (Lat) & 215.7 & 183 & - & - & - & - & 275 \\
Hip (AP) & 686.9 & 177 & - & - & - & - & 300 \\
\hline
\end{tabular}

\section{Discussions}

This study evaluates the dose received by adult patients who underwent X-rays examinations in seven (7) radiographic rooms in Senegal. Great variations in patient doses were found in this survey as shown by the boxplots (Figure 3 and Figure 4) in term of ESD and DAP. Some reasons for the variations were evidenced. For instance, a wide range was observed for tube load and tube voltage (Table 2) for all the examinations. For chest radiography, the loading factors extended from 2.2 to $80 \mathrm{mAs}$ and 55 to $128 \mathrm{kVp}$. The use of high tube voltage increases the penetration of the beam and should be accompanied with low tube load to reduce the dose to the patient. Honey et al. (2005) reported that an optimum image quality can be obtained with computed radiography for chest examination in the $75-90 \mathrm{kVp}$ range rather than higher energies which are mostly used in clinical routine [26]. Samei et al. (2005) recommend additional filtration rather than low tube voltage for chest examination [27]. It should be noticed that the energy range for chest imaging depends on the technology of the detector. The entrance surface doses obtained for all the examinations have a wide range of variation: $0.57-34.12 \mathrm{mGy}$ and $0.97-46.17 \mathrm{mGy}$ for lumbar spine (AP and Lat), $0.46-10.47 \mathrm{mGy}$ and $0.71-42.81 \mathrm{mGy}$ for thoracic spine (AP and Lat), $0.21-9.29 \mathrm{mGy}$ for cervical spine AP and $0.38-8.13 \mathrm{mGy}$ for abdomen AP. These variations show the need to harmonize imaging procedures and can be attributed to different X-rays units, exposure factors, image receptors and most importantly, variations in patient sizes. In addition to the tube-defined exposure parameters, other equipment-related, technologically limited factors also affect the dose to the patient. They are three-phase generators, insufficient beam filtration and manual exposure adjustment. The higher the maximum/minimum ratios of the ESD values, the higher the potential for reducing the dose. Inherent tube filtration in diagnostic radiology is $2.5 \mathrm{~mm}$ of equivalent aluminum. Additional filtration in the X-ray beam can be used to remove the low energy part of the spectrum which is totally absorbed by the patient without contributing to image quality. Kawashima et al. (2017) reported a reduction of ESD up to $40 \%$ without degrading the quality of the image when using additional $\mathrm{Cu}$ filters after simulating $20 \mathrm{~cm}$ acrylic phantom [28]. Increasing the FFD also can lead to a 
dose reduction for all the examinations. Regarding the DAP, the two sigma range (95\% Confidence Interval) shows the same variations than the ESD values. Table 4 and Table 5 show a comparison of dose audit in Senegal (this work) and some in other countries in terms of ESD and DAP. The third quartile of the entrance surface dose presented here are generally lower than the results of other surveys. However, for thoracic AP and Pelvis AP, our $3^{\text {rd }}$ quartiles are respectively $38 \%$ and $16 \%$ higher than their corresponding values. In terms of dose-area product, most of our values are higher than those of other surveys; these large values can be explained by the collimation because the irradiated area is proportional to the dose area product. The recent recommendations favor median values for setting DRLs and go to the 75th percentile and in this study, the median values are much lower than their corresponding DRLs for all the examinations.

The manual setting of the exposure parameters affects mostly the patient dose; the high standard deviation is the confirmation. The new technologies such as flat panel detector (Digital radiography with Automatic Exposure Control) can be an issue for dose reduction but require adjustment of exposure parameters and radiographer's skills. The use of AEC can reduce the collimation to the anatomical district of interest, resulting in a smaller irradiated area, lower organ dose and sparing more tissues that are not of interest. The scattered radiation is proportional to the collimation and could degrade the subject contrast without carrying information to the detector [29]. Reducing the field of view (FOV) to the area of interest could reduce the scattered radiation and the dose received by other organs as well as improve image quality. The use of anti-scatter grid, post processing algorithms (window level and width), specific training for technologists and radiologists, and implementation of quality assurance program can lead to dose reduction and improvement of the image quality [30]. The local dose audit reported in this study reveals the practice in few X-ray units. Although it is not representative of what happens in every hospital, it is an indication that dose optimization is possible in Senegal. The differences can be due to the effect that the diagnostic reference levels available for comparison are of $\mathrm{Eu}$ ropean origin which have not been determined with the same equipment, training of machine users and patient morphology but also used a large sample.

The DRLs play critical role in the optimization of radiation dose. The great variations in dose found between medical centers indicate the necessity to optimize the practices. Our results can be applied to meet some requirement for the establishment of NDRLs which in turn can help to prevent unnecessary radiation dose. Low doses are critical because of their stochastics effect at long term. That is why it is necessary for each institution to develop protocols for dose measurement that could contribute to both the establishment of LDRLs as well as in the evaluation of the local radiographic practice.

\section{Conclusion}

The survey has been conducted to investigate patient doses for nine routine 
types of X-ray examinations in radiology. The exposure of 2217 patients was analyzed; entrance surface dose and dose area product were evaluated. This study indicates the need to standardize the medical X-ray examination technique. It is very important to perform real patient dose measurements in hospitals. In that sense, the survey results are the link between patient dosimetry, as the first step in optimization of radiation protection and quality assurance program in diagnostic radiology. It is of great importance to extend the survey to a larger number of hospitals in order to establish diagnostic reference levels at the national level. Reference dose levels for diagnostic radiology examination provide the benchmark for comparing X-rays exposures from different facilities, to reduce patient doses and maintain good image quality with respect to basic principles of radiation protection of patient (justification and optimization). It is expected that this survey will encourage further efforts in organizing radiation protection program to effectively monitor patient dose and optimize the radiology practice.

\section{Declarations}

The research did not receive any specific grant from funding agencies in the public, commercial or not-for-profit sector.

\section{Acknowledgements}

We would like to thank Professor Fernando Ferroni (INFN) and Professor Marco Silari (CERN) for reading this manuscript and Dr. Modou Mbaye (Cheikh Anta Diop University of Dakar) for his assistance during the analysis of the data.

\section{Conflicts of Interest}

The authors declare that they have no conflicts of interest.

\section{References}

[1] Recommendations of the ICRP (1991) The System of Protection for Proposed and Continuing Practices. Annals of the ICRP, 21, 32-49. https://doi.org/10.1016/0146-6453(91)90069-S

[2] International Atomic Energy Agency (2018) Be Informed about the Safe Use of Ionizing Radiation in Medicine. Information to Help Health Professionals Achieve Safer Use of Radiation in Medicine for the Benefit of Patients. https://rpop.iaea.org/RPOP/RPoP/Content/index.htm

[3] Harding, K. and Thomson, W.H. (1997) Radiological Protection and Safety in Medicine-ICRP 73. European Journal of Nuclear Medicine, 24, 1207-1209.

[4] Rosenstein, M. (2008) Diagnostic Reference Levels for Medical Exposure of Patients: ICRP Guidance and Related ICRU Quantities. Health Physics, 95, 528-534. https://doi.org/10.1016/0146-6453(91)90069-S

[5] (2014) The European Basic Safety Standards (BSS) for Protection against Ionising Radiation, Official Journal of European Union. http://eur-lex.europa.eu/JOHtml.do?uri=OJ:L:2014:013:SOM:EN:HTML

[6] (1999) Radiological Protection 109. 
https://ec.europa.eu/energy/sites/ener/files/documents/109_en.pdf

[7] Konate, I., Monnehan, G.A., Gogon, D.B.L.H., Dali, T.P.A., Koua, A.A. and Djagouri, K. (2019) Diagnostic Reference Level in Frontal Chest X-Ray in Western Côte d'Ivoire. World Journal of Nuclear Science and Technology, 9, Article No. 4.

[8] Moifo, B., Tapouh, J.R.M., Guena, M.N., Ndah, T.N., Samba, R.N. and Simo, A. (2017) Diagnostic Reference Levels of Adults CT-Scan Imaging in Cameroon: A Pilot Study of Four Commonest CT-Protocols in Five Radiology Departments. Open Journal of Medical Imaging, 7, Article No. 1. https://doi.org/10.4236/ojmi.2017.71001

[9] Evaluation of Patient Doses from Common Radiographic Procedures in Selected Hospitals in Baguio City. https://cdu.herdin.ph/index.php/component/herdin/?view=research\&cid=40272

[10] Le Heron, J. (1999) Guidelines on Patient Dose to Promote the Optimisation of Protection for Diagnostic Medical Exposures: Documents of the NRPB v 10(1), 1999. Radiation Protection News and Notes, No. 46, 17-18.

[11] Petoussi-Henss, N., Zankl, M., Drexler, G., Panzer, W. and Regulla, D. (1998) Calculation of Backscatter Factors for Diagnostic Radiology Using Monte Carlo Methods. Physics in Medicine \& Biology, 43, 2237-2250. https://doi.org/10.1088/0031-9155/43/8/017

[12] European Commission (1996) European Guidelines on Quality Criteria for Diagnostic Radiographic Images: Eur 16260 EN.

[13] ICRU (n.d.) ICRU Report 74, Patient Dosimetry for X Rays Used in Medical Imaging.

https://www.icru.org/report/patient-dosimetry-for-x-rays-used-in-medical-imaging -report-74

[14] Mohammedzein, T.S. (2009) Measurements of Radiation Dose to Patients Undergoing Some Common Radiographic X-Ray Examinations in Wad Madani Hospitals. 41.

[15] Compagnone, G., Pagan, L. and Bergamini, C. (2005) Local Diagnostic Reference Levels in Standard X-Ray Examinations. Radiation Protection Dosimetry, 113, 54-63. https://doi.org/10.1093/rpd/nch432

[16] Nickoloff, E.L., Lu, Z.F., Dutta, A.K. and So, J.C. (2008) Radiation Dose Descriptors: BERT, COD, DAP, and Other Strange Creatures. RadioGraphics, 28, 1439-1450. https://doi.org/10.1148/rg.285075748

[17] (1992) National Protocol for Patient Dose Measurement in Diagnostic Radiology, Dosimetry Working Party of the Institute of Physical Sciences in Medicine, Natioanl Rdiological Protection Board.

[18] Hart, D., Hillier, M.C. and Wall, B.F. (2002) Doses to Patients from Medical X-Ray Examinations in the UK 2000 Review. United Kingdom, 08-595148-5-4.

[19] Internationale Atomenergie-Organisation (2007) Dosimetry in Diagnostic Radiology: An International Code of Practice. International Atomic Energy Agency, Vienna.

[20] Hart, D., Hillier, M.C. and Wall, B.F. (2005) Doses to Patients from Radiographic and Fluoroscopic X-Ray Imaging Procedures in the UK-Review. 95.

[21] IRSN PRP-HOM (2020) Analyse des donnees relatives a la mise a jour des niveaux de reference diagnostiques en radiologie et en medecine nucleaire. Bilan 2016-2018. Rapport parut en 2020.

[22] Schegerer, A.A. (2016) KVB-RQ-Radiologie-Strahlenschutz-Diagnostische Refe- 
renzwerte-20031.pdf. Federal official Journal, 22, 2010.

[23] (2000) The Swedish Radiation Protection Institute's Regulations on Xray Diagnostics.

[24] European Commissi (2014) Radiation Protection No. 180. https://ec.europa.eu/energy/sites/ener/files/documents/RP180\%20part2.pdf

[25] (n.d.) Niveaux de référence diagnostiques (NRD) en radiologie par projection. https://www.astrm.ch/files/Dokumente/Verband/Fachstellen/Strahlenschutz/R-06-0 4_niveaux_de_reference_diagnostiques_NRD_en_radiologie_par_projection.pdf

[26] Honey, I.D., Mackenzie, A. and Evans, D.S. (2005) Investigation of Optimum Energies for Chest Imaging Using Film-Screen and Computed Radiography. The British Journal of Radiology, 78, 422-427. https://doi.org/10.1259/bjr/32912696

[27] Samei, E., Dobbins, J.T., Lo, J.Y. and Tornai, M.P. (2005) A Framework for Optimising the Radiographic Technique in Digital X-Ray Imaging. Radiation Protection Dosimetry, 114, 220-229. https://doi.org/10.1093/rpd/nch562

[28] Kawashima, H., Ichikawa, K., Nagasou, D. and Hattori, M. (2017) X-Ray Dose Reduction Using Additional Copper Filtration for Abdominal Digital Radiography: Evaluation Using Signal Difference-to-Noise Ratio. Physica Medica, 34, 65-71. https://doi.org/10.1016/j.ejmp.2017.01.015

[29] Bushberg, J.T. (1998) The AAPM/RSNA Physics Tutorials for Resident. X-Ray Interactions. RadioGraphics, 18, No. 2.

https://doi.org/10.1148/radiographics.18.2.9536489

[30] Rill, L.N., Brateman, L. and Arreola, M. (2003) Evaluating Radiographic Parameters for Mobile Chest Computed Radiography: Phantoms, Image Quality and Effective Dose. Medical Physics, 30, 2727-2735. https://doi.org/10.1118/1.1611291 